\title{
Diatomite inspired nanostructured quartz as a piezoelectric material
}

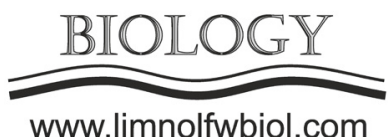

\author{
Aggrey P. ${ }^{1}$, Salimon A.I. ${ }^{1}$, Korsunsky A.M. ${ }^{1,2}$ \\ ${ }^{1}$ Hierarchically Structured Materials lab Center for Energy Science and Technology, Skolkovo Institute of Science and Technology, \\ Moscow, Russia \\ ${ }^{2}$ MBLEM, Department of Engineering Science, University of Oxford, Oxford OX1 3PJ, UK
}

\begin{abstract}
Diatomite continues to fascinate the scientific community with widespread applica tion in the energy sector. However, these applications have not encompassed all energy segments. Herein, we report a soft chemistry approach to synthesize nanostructured quartz from diatomite for piezoelectric applications. The synthesis parameters will be varied to understand the effects on the structure and formation of quartz. Preliminary results confirm that diatomite is a siliceous material with a neat nanoscale morphology. The formation of nanostructured quartz from diatomite will expand the range of applications of diatomite in energy related applications.
\end{abstract}

Keywords: diatomaceous earth, nanoporous, energy, dissolving and precipitating, quartz transformation

\section{Introduction}

Naturally formed through the build-up of amorphous silica cell walls of dead diatoms in marine sediments which ultimately transform to sedimentary rock diatomite over geological epochs, diatoms have inspired the diatom bionanotechnology, a new development in scientific research. Recent reports of a vast application of diatomaceous earth (DE) in energy harvesting and storage applications indicates that diatomite could potential be the next energy material. At present, applications in batteries, supercapacitors, thermal energy storage, thermoelectric generators and photovoltaics have been reported (Karaman et al., 2011; Campbell et al., 2016; Yang et al., 2018; Zong et al., 2018). Although it is rich source of silica, which can be transformed to quartz, for piezoelectric applications, there are currently no reports of such applications. Diatomite, considering it's desirable neatly formed nanoporous structure is a suitable precursor for nanostructured quartz. The piezoelectric effect in materials is known to increase at nanoscale. More precisely, pores have been reported to increase the piezoelectric effect in materials (Su et al., 2019). This confirms the suitability of diatomite as a silica precursor for nanostructured quartz.

Naturally occurring diatomite is made up of mainly Opal (SiO2. nH2O) and minor quantities quartz and other minerals. Opal is amorphous, making naturally occurring diatomite an almost perfect amorphous material. Conventional routes of obtaining quartz from amorphous silica involve high temperature synthesis, and this is not suitable for the neatly formed nanoporous structure of diatomite. Although, the dissolution and precipitation of silica in acidic and basic media have been widely studied (Bertone et al., 2003; Carretero-Genevrier et al., 2013; Buckley et al., 2018), these studies have not been extended massively to naturally occurring diatomite. Knowing very well it is a siliceous material, it is clear that a similar dissolution and precipitation mechanism will be observed in diatomite. However, the effects of such mechanisms on the neatly formed nanoporous structure is yet to be revealed. Since diatomite is highly desired due to its neat nanoscale structure, it is necessary to design a protocol capable of dissolving and precipitating silica without significant damage to the parent structure.

\section{Material and Methods}

A yet to be determined mass of diatomite powder will be added to $100 \mathrm{ml}$ of $\mathrm{NaOH}$ and heated to between $200-300{ }^{\circ} \mathrm{C}$ for 2 hours, with a heating rate of $6{ }^{\circ} \mathrm{C} /$ min. The system will be quenched to $70{ }^{\circ} \mathrm{C}$ for $10-15$ minutes in water circulation. The powder obtained will be purified via dialysis, maintaining a $\mathrm{pH}$ of 8 . The obtained powder will be analysed using XRDA, SEM, Atomic Force Microscopy (AFM), Raman Spectroscopy, BET porosimeter, and Powder particle size Analyser. The synthesis parameters will be varied to understand the effects on the structure and formation of quartz.

\section{Results and Discussion}

The preliminary results obtained from Scanning Electron Microscopy (SEM) revealed the nanoscale

*Corresponding author.

E-mail address: patrick.aggrey@skoltech.ru (P. Aggrey)

(C) Author(s) 2020. This work is distributed under the Creative Commons Attribution 4.0 License. 
structure of raw diatomite. The major phase in the raw powder is Opal, accompanied with a minor quantity of quartz confirmed through X-ray Diffraction Analysis (XRDA). The powder particles size ranges between 1-10 $\mu \mathrm{m}$ as measured on the Fritsch Particle Analyzer. The specific surface area of the diatomite powder was $20.4306 \mathrm{~m}^{2} / \mathrm{g}$, measured using the Quantachrome Nova 2200e BET porosimeter.

\section{Conclusion}

The choice of diatomite as a precursor for the formation of nanostructured quartz is as a result of its naturally formed neat nanoporous structure. This feature is confirmed by preliminary powder characterisation carried out. The formation of nanostructured quartz from diatomite and the understanding of the effects of synthesis parameters will open a range of opportunities and require efficient techniques to help control the morphology of the precursor diatomite.

\section{Acknowledgement}

The authors wish to acknowledge the Center for Energy Science and Technology (CEST) at Skoltech.

\section{References}

Bertone J.F., Cizeron J., Wahi R.K. et al. 2003. Hydrothermal synthesis of quartz nanocrystals. Nano Letters 3(5): 655-659. DOI: 10.1021/nl025854r
Buckley P., Hargreaves N., Cooper S. 2018. Nucleation of quartz under ambient conditions. Communications Chemistry 1(1): 1-10. DOI: 10.1038/s42004-018-0049-4

Campbell B., Ionescu R., Tolchin M. et al. 2016. Carboncoated, diatomite-derived nanosilicon as a high rate capable Li-ion battery anode. Scientific Reports 6. DOI: 10.1038/ srep33050

Carretero-Genevrier A., Gich M., Picas L. et al. 2013. Softchemistry-based routes to epitaxial $\alpha$-quartz thin films with tunable textures. Science 340(6134): 827-831. DOI: 10.1126/ science. 1232968

Karaman S., Karaipekli A., Sarı A. et al. 2011. Polyethylene glycol (PEG)/diatomite composite as a novel form-stable phase change material for thermal energy storage. Solar Energy Materials and Solar Cells 95(7): 1647-1653. DOI: 10.1016/j.solmat.2011.01.022

Su Y.L., Gupta K., Hsiao Y.L. et al. 2019. Gigantic enhancement of electricity generation in piezoelectric semiconductors by creating pores as a universal approach. Energy \& Environmental Science 12(1): 410-417. DOI: 10.1039/C8EE02081G

Yang Y., Li A., Cao X. et al. 2018. Use of a diatomite template to prepare a MoS 2/amorphous carbon composite and exploration of its electrochemical properties as a supercapacitor. RSC Advances 8(62): 35672-35680. DOI: 10.1039/C8RA07062H

Zong P.A., Makino D., Pan W. et al. 2018. Converting natural diatomite into nanoporous silicon for eco-friendly thermoelectric energy conversion. Materials \& Design 154: 246-253. DOI: 10.1016/j.matdes.2018.05.042 